\title{
Anabases
}

ANABASES Traditions et réceptions de l'Antiquité

$10 \mid 2009$

Varia

\section{Il maestro, il discepolo e gli altri maestri : un percorso nella storia romana}

Andrea Giardina

\section{(2) OpenEdition}

Journals

Edizione digitale

URL: http://journals.openedition.org/anabases/631

DOI: 10.4000/anabases.631

ISSN: 2256-9421

Editore

E.R.A.S.M.E.

\section{Edizione cartacea}

Data di pubblicazione: 1 ottobre 2009

Paginazione: 61-73

ISSN: 1774-4296

\section{Notizia bibliografica digitale}

Andrea Giardina, « II maestro, il discepolo e gli altri maestri : un percorso nella storia romana »,

Anabases [Online], 10 | 2009, Messo online il 01 octobre 2012, consultato il 21 octobre 2019. URL

http://journals.openedition.org/anabases/631 ; DOI : 10.4000/anabases.631 
Anabases 10 (2009), p. 61-73.

\section{Il maestro, \\ il discepolo e gli altri maestri: un percorso nella storia romana}

Andrea Giardina

Per chi abbia intrapreso gli studi universitari verso la fine degli anni Sessanta, affrontare il tema proposto da Anabases comporta un duplice impegno. Ricordare e ripensare le proprie esperienze, cercare di comunicarle senza cadere nell'autobiografismo e nel compiacimento, far emergere gli elementi che possano essere d'interesse più generale, tutto questo non basta. Negli ultimi quarant'anni il nostro habitat universitario è mutato più volte, per sollecitazioni leggere e ripetute oppure per scosse traumatiche, con il risultato che se per incanto fossimo riportati a quegli anni, scopriremmo sgomenti di aver smarrito la parola, come quegli emigranti che tornati al loro paese parlano un dialetto arcaico o una lingua imbastardita. E' dunque necessario collocare quelle esperienze entro le trasformazioni e le crisi più ampie che ne hanno rappresentato il contesto.

Eravamo studenti in un'università che traeva la sua organizzazione, i suoi modelli di comportamento e i suoi valori da una tradizione antica, per taluni aspetti ottocentesca, per altri molto più remota. In Italia, il corpo docente era limitato, tranne qualche eccezione, ai professori ordinari, mentre gli assistenti non avevano obblighi didattici (erano solitamente dissuasi dal tenere lezioni o esercitazioni di qualunque genere); nei consigli di facoltà sedevano i soli professori ordinari; non esistevano dipartimenti ma «istituti», che in molti casi erano costituiti da una sola cattedra; non c'erano dottorati di ricerca; le biblioteche, anch'esse d'istituto, erano finalizzate alle esigenze di studio dei professori, e la presenza degli studenti era scoraggiata, sia per mancanza di personale sia per motivi di opportunità; i professori erano personaggi algidi e spesso temuti, cui non si accedeva con facilità; il pubblico che assisteva alle lezioni era silenzioso come un'assemblea spartana e il dialogo con il docente (quando si verificava) era interpersonale; nelle lezioni si assumevano come scontate tutte le informazioni di base, date per acquisite nel corso dell'apprendimento scolastico; i seminari erano rari o inesistenti; 
considerato il basso numero di prove da sostenere, i programmi di esame erano a volte vastissimi e potevano comprendere migliaia di pagine, spesso altamente specialistiche.

Come tutte le generalizzazioni, questo quadro può risultare discutibile perché non rappresenta la varietà delle situazioni, perché non tiene conto del fatto che ambiti disciplinari diversi potevano determinare comportamenti diversi, perché trascura le eccentricità individuali e la forza di deriva dei singoli temperamenti. I colleghi che avranno difficoltà a inserirvi la propria personale esperienza non dovrebbero tuttavia negarne l'autenticità sociologica ${ }^{1}$.

Nel sistema alquanto complicato delle facoltà di lettere, la storia romana, ovvero la disciplina cui avrei dedicato in futuro la mia vita professionale, occupava una posizione speciale. Tra le cinque materie dette «fondamentali comuni» perché tutti gli studenti della facoltà erano indistintamente tenuti a sostenerne l'esame, due erano discipline di ambito antichistico, la letteratura latina e la storia romana. Valeva dunque il principio secondo il quale uno studente poteva conseguire una laurea in lettere senza aver sostenuto gli esami di storia medievale, di storia moderna o di storia contemporanea, ma non senza che le sue conoscenze di storia romana fossero state esaminate e approvate. La storia romana era dunque la regina delle discipline storiche e insieme parte essenziale tra le misure atte a giudicare la validità di una cultura di base. Intorno al 1966, quando cominciavo a frequentare i corsi di lettere classiche, nessuno, tra gli studenti, sembrava porsi domande sulle ragioni di un simile primato: esso appariva al contrario come un dato naturale, che stabiliva per altro una perfetta simmetria con l'autorevolezza indiscussa del liceo classico, da cui provenivano in massima parte i ceti dirigenti del paese (questo liceo era l'unico che consentisse l'accesso a tutte le facoltà universitarie, anche quelle scientifiche, mentre lo stesso non poteva dirsi del liceo scientifico). La circostanza che un simile primato fosse almeno in parte riconducibile al culto fascista della romanità, che aveva inevitabilmente favorito l'autonomia della storia romana rispetto alla storia greca (così come della storia dell'arte romana rispetto alla greca) ${ }^{2}$ era ritenuta irrilevante o comunque passava sotto silenzio. Non approfondisco, perché esula dalle mie esperienze formative di allora, il ruolo svolto parallelamente, con risalto per molti aspetti ancora maggiore, dalle materie romanistiche nelle facoltà di giurisprudenza.

Era questa l'atmosfera in cui avvenne l'incontro con il mio futuro maestro Santo Mazzarino. Alla metà degli anni Sessanta, il prestigio di questo studioso era grandissimo. Nato nel 1916, era un uomo ancora giovane, nel pieno delle sue energie intellettuali. Si era trasferito dall'università di Catania, sua città natale, alla Sapienza di Roma nel 1964, per succedere ad Aldo Ferrabino. La sua fama dipendeva in primo luogo dalla

1 Per un quadro d'insieme, cf. p. es. A. SAntoni Rugiu, Chiarissimi e Magnifici. Il professore nell'università italiana dal 1700 al 2000, La Nuova Italia, Firenze 1991.

2 Cf. A. Giardina, s.v. "Archeologia”, in V. de Grazia e S. Luzzatto (a cura di), Dizionario del fascismo, 1, Einaudi, Torino, 2002, p. 86-90. 
quantità e dall'importanza delle sue opere e dal fatto che esse coprissero con pari fecondità di risultati l'intero arco della storia antica ${ }^{3}$. Al suo primo libro, Stilicone. La crisi imperiale dopo Teodosio (Istituto italiano per la storia antica, Signorelli, Roma 1942) ${ }^{4}$, derivata dalla tesi di laurea discussa nel 1936 sotto la guida di Luigi Pareti, avevano fatto seguito, oltre a decine di saggi minori, volumi come Dalla monarchia allo stato repubblicano. Ricerche di storia romana arcaica (Agnini, Catania 1945) ${ }^{5}$, Fra Oriente e Occidente. Ricerche di storia greca arcaica (La Nuova Italia, Firenze 1947) ${ }^{6}$, Introduzione alle guerre puniche (Crisafulli, Catania 1948) ${ }^{7}$, Aspetti sociali del quarto secolo. Ricerche di storia tardo-romana (L'Erma di Bretschneider, Roma 1951$)^{8}$, Storia romana e storiografia moderna (Conte, Napoli 1954), L'impero romano (vol. II del Trattato di storia romana scritto con G. Giannelli, Tumminelli, Roma 1956) ${ }^{9}$, La fine del mondo antico (Bompiani, Milano 1959) ${ }^{10}$, Il pensiero storico classico (Laterza, Bari 1966).

A questa fama d'autore si univa, con un effetto moltiplicatore, una sorta di mitologia legata all'uomo: come molti grandi maestri, Santo Mazzarino è stato anche un "personaggio", consapevole di esserlo e interprete egli stesso della propria maschera (forse anche per questo gli piaceva ricordare le parole che secondo Svetonio Augusto avrebbe pronunciato in punto di morte: «Ho rappresentato bene questo mimo della mia vita? Se vi è piaciuto il giuoco, date il vostro plauso, e tutti con gioia accompagnatemi») ${ }^{11}$. Questa mitologia si fondava su due caratteristiche strettamente collegate: una cultura talmente vasta e profonda che sembrava sfiorare l'onniscienza, una memoria fuori del

3 Per le informazioni sulla carriera scientifica e accademica di Mazzarino rinvio al Nachruf da me pubblicato su Gnomon 62 (1990), p. 374-379.

4 Seconda edizione, Rizzoli, Milano, 1990, con una mia introduzione intitolata Stilicone o l'antico destino degli uomini vinti; sull'attualità di quest'opera cf. anche F. Tessitore, Mazzarino e lo storicismo degli storici, Università degli studi di Catania, Facoltà di Lettere e Filosofia, Catania, 2003, p. 76-78.

5 Seconda edizione, Rizzoli, Milano, 1992, con introduzione di A. FraschetTI.

6 Seconda edizione, Rizzoli, Milano, 1989, con introduzione di F. Cassola. Questa edizione presenta un'importante appendice, contenente una risposta inedita dell'autore ("Per un discorso sul metodo", p. 405-415) alla recensione pubblicata nel 1948 da A. Momigliano (p. 397-403). L'opera ha avuto una recente ristampa (Boringhieri, Torino 2007). - Per un ripensanemento in parallelo della figura dei due studiosi, M. MAzzA, "Ricordo di Santo Mazzarino e di Arnaldo Momigliano” Orpheus n.s., 12 (1991), p. 317-341.

7 Seconda edizione, Rizzoli, Milano, 2003, con introduzione di D. Musti.

8 Seconda edizione, Rizzoli, Milano 2002, con introduzione di E. Lo CASCIO; in appendice (p. 379-423), la ristampa di un saggio del 1981, "Sull'epigrafe dioclezianea di Afrodisiade "Bicharactam»: per l'interpretazione romana delle misure «inflattive»". Cf. anche L. Cracco Ruggini, "La società tardoantica secondo Santo Mazzarino", Rivista storica italiana 101 (1989), p. 122-147.

9 Il volume scritto da Mazzarino ha avuto una fortuna autonoma, a partire dal 1973, con gli editori Laterza e il titolo L'impero romano.

10 Seconda edizione, Rizzoli, Milano, 1988; ristampa Boringhieri, Torino, 2008.

11 Cito dalla traduzione di MAZZARINo, L'impero romano..., p. 95. 
comune. Agli allievi e ai colleghi questo studioso dava la sensazione di sapere tutto e di ricordare tutto, ed è bene precisare che queste doti non si limitavano all'ambito pur immenso della storia antica. Durante le lezioni universitarie o le conferenze citava a memoria interminabili pezzi di autori greci e latini: non si aveva l'impressione di un ricordo facile e automatico ma di uno sforzo mirante a ricostruire la forma di un testo da lui studiato magari anni, se non decenni prima. Come il rabdomante cerca le vene sotterranee di acqua e di metalli, così Mazzarino sembrava attingere depositi di parole, correnti di testi, giacimenti sperduti di segni.

Queste doti concorrevano a determinare una posizione dominante. Oggi, la disponibilità di autori e di documenti online o su supporti informatici ha reso accessibile teoricamente a tutti la possibilità di reperire riscontri e paralleli testuali. Allora, la differenza era data unicamente dalla performance individuale, e Mazzarino era solito stupire i lettori e gli ascoltatori «versando nel dibattito" (così soleva dire ricorrendo a una metafora eloquente) documenti mai considerati prima. Il procedimento si attuava mettendo in relazione tipi diversi di documenti, per esempio testi letterari ed epigrafici che gli specialismi avevano reciprocamente oscurato, oppure documenti di epoche diverse, a volte molto lontane tra loro.

Una simile figura di studioso era, per taluni aspetti, il prodotto del sistema universitario nel quale si era formata, ma aveva caratteristiche tali che la rendevano eccezionale, fuori misura, anomala. Lo stesso termine «studioso» appare decisamente riduttivo, trattandosi in verità di un intellettuale che aveva prospettive originali su molti argomenti del pensiero filosofico moderno e sulla politica contemporanea (il suo ultimo scritto, pubblicato pochi giorni dopo la morte, è una riflessione, che oggi alcuni potrebbero giudicare profetica, sulla riforma del sistema elettorale italiano e sulle motivazioni delle masse in una democrazia contemporanea) ${ }^{12}$. La cultura antichistica del Novecento ha avuto alcuni grandi maestri, molti storici di primo piano, moltissimi bravi storici, ma pochi autentici intellettuali, e Mazzarino è stato al tempo stesso un grande maestro e un intellettuale. Forse anche per questo egli soleva ripetere di non aver avuto veri maestri: del professore che fu suo relatore di tesi, Luigi Pareti, non parlava mai, e le espressioni

12 Lo scritto, datato undici giorni prima della sua scomparsa, avvenuta il 18 maggio del 1987, fu pubblicato su la Repubblica, Roma 2 giugno 1987, e successivamente su Rinascita del 6 giugno 1987. Per una valutazione d'insieme del rapporto tra storia antica e storia contemporanea in Mazzarino, cf. G. GIARrizzo, "Mazzarino: storici antichi e storiografia moderna", in Omaggio a Santo Mazzarino, un maestro, Università degli studi di Catania, Facoltà di Lettere e Filosofia, Catania 2003, specialmente p. 17: «Il presente poneva questioni, cui il passato antico riletto attraverso il prisma della sua storiografia doveva fornire risposte che chiamavano in causa a lor volta gli storici moderni (o modernissimi).» Per Mazzarino e lo storicismo del secondo Novecento, cf. soprattutto F. Tessitore, Mazzarino e lo storicismo degli storici... 
da lui messe per iscritto a proposito di questo rapporto rivelano alcune ambiguità molto significative ${ }^{13}$.

Posto a confronto da un lato con quella realtà universitaria, dall'altro con una simile personalità di maestro, uno studente poteva facilmente sentirsi sopraffatto ed essere tentato dalla ricerca di vie più rassicuranti. Non so quanto, nel caso mio come in quello di altri allievi romani di Santo Mazzarino in quegli anni (del Mazzarino catanese nulla potrei dire se non di seconda mano $)^{14}$, si possa parlare di un'autentica scelta, lucida e ragionata, a favore della storia romana: probabilmente siamo stati noi «scelti» da un complesso di circostanze e di fattori umani, sedotti dal privilegio di un contatto con un maestro ritenuto dai più inarrivabile, attratti dalla mutevolezza di un uomo che alternava austera severità e tenerezza paterna, grati per la sua generosità, soddisfatti da un precoce senso di appartenenza a una «scuola» autorevole ${ }^{15}$. Solo così riesco oggi a spiegarmi la determinazione con cui ho svolto, tra i diciannove e i venti anni, una tesi di laurea su un argomento quale "I criteri di datazione delle leggi del Codice Teodosiano" (Mazzarino mi ripeteva: «è la più bella tesi che abbia mai assegnato», ma ho il fondato sospetto che dicesse la stessa cosa ad altri...) ${ }^{16}$. Ricordo che quando gli chiesi indicazioni bibliografiche per avviare il lavoro, mi rispose: «La Sua è una tesi sul basso impero, quindi tutta la bibliografia sul basso impero. Cominci da Costantino, tutta la bibliografia su Costantino...".

L'esperienza è stata molto formativa, e ancora oggi credo di trarne vantaggi, ma non c'era nulla, in quel tema, che potesse entusiasmare un giovane e portarlo nel vivo della passione e della riflessione storica. Prevaleva su tutto la volontà di suscitare l'approvazione del maestro. Questi risvolti psicologici, volitivi, questo viluppo di ambizione,

13 A. Giardina, "Stilicone o l'antico destino degli uomini vinti...”, p. IX.

14 Cf. ora G. Giarrizzo, "Le lezioni di un maestro. Un commento, un incontro, un ricordo", Mediterraneo antico 9 (2006), p. 107-151. - Gli allievi romani cui mi riferisco, che si sono laureati con Santo Mazzarino in quegli anni all'Università di Roma La Sapienza e hanno intrapreso successivamente la carriera universitaria, sono, oltre a me, Luciano Camilli, Augusto Fraschetti, Elio Lo Cascio.

15 Per molti di questi aspetti, cf. F. WAQUET, Les enfants de Socrate. Filiation intellectuelle et transmission du savoir, XVII $-X X I^{e}$ siècle, Albin Michel, Paris, 2008 (p. es. p. 117-118: "L'honneur d'être élève» e il cap. 5, «Filiation»); sul senso debole della parola «scelta» in questo genere di relazioni, cf. in particolare p. 60-67, "Les critères du choix», e specialmente p. 66: «L'expression "relation choisie" laisserait entendre une détermination rationnelle et volontaire qu'il convient de tempérer.»

16 Avrei dovuto riscrivere, sotto forma di libro, i Regesten di Otto SEECK (Stuttgart 1919). L'utilità di una simile impresa complessiva mi appare oggi assai dubbia. La mia esperienza su questo tema è confluita tuttavia in due lavori giovanili ["L'epigrafe di Iunius Bassus ad Aqua Viva e i criteri metodici di Godefroy”, Helikon 11-12 (1971-1972), p. 253-278 e "Sul problema della fraus monetae", ivi, 13-14, 1973-1974, p. 184-190] e nella pubblicazione, con F. GReLle, dell'epigrafe da Trinitapoli contenente parte del testo di una costituzione di Valentiniano I ["La Tavola di Trinitapoli: una nuova costituzione di Valentiniano I”, Mélanges de l'École française de Rome, Antiquité 95 (1983), p. 249-303]. 
orgoglio e sentimento, sono aspetti tipici e non trascurabili nella storia delle scuole universitarie e dovremmo rifuggire dal dissolverli sempre e comunque in specifiche problematiche culturali. Quando l'ingresso in una "scuola» è molto precoce, le ragioni culturali sono mere suggestioni e giustificano l'appartenenza soltanto ex post.

Il documento suggerito da Anabases invita anche a riflettere sulla validità del termine «discepolo», che in italiano suona certamente più arcaico del francese «disciple». Ritengo che esso qualifichi perfettamente il mio rapporto con Santo Mazzarino soprattutto se si tengono presenti le due caratteristiche principali del campo semantico del termine latino discipulus: la parola rimanda infatti primariamente alla sfera del discere, dell'apprendimento, dell' 'istruzione, dell'educazione, ma su questo significato $s^{\prime}$ 'innesta quasi automaticamente quello di obbedienza, regola di condotta, disciplina ${ }^{17}$. Nell'idealtipo mazzariniano del discepolo, che io ho cercato di interpretare al meglio delle mie possibilità intellettive e del mio autocontrollo psicologico, l'apprendimento e la costruzione di una personalità di studioso erano inscindibili dal rispetto di ben precise regole di condotta, che si misuravano sia nel campo della formazione e della produzione scientifica (conoscenza totale della materia, modestia, probità, originalità, completezza dell'informazione, rapporto inscindibile tra citazione e autopsia, ecc.) sia nel campo dei comportamenti accademici. E' inutile precisare quanto questi ultimi si esprimessero anche in una certa cautela e in un visibile senso della misura nei rapporti con le altre scuole e in particolar modo con quelle ritenute meno vicine o ostili (la parola «fedeltà» aveva un suo peso, come il suo contrario «tradimento») ${ }^{18}$. Da queste considerazioni discende la consapevolezza di essere stato un «discepolo» di Santo Mazzarino. Ma con la precisazione che la qualifica di «maestro» - per i motivi che ho appena esposto - non genera automaticamente quella di «discepolo». Come avrò modo di dire fra poco, ho avuto altri maestri (anche se nessuno così decisivo e importante come Santo Mazzarino) ma di nessun altro sono stato discepolo.

L'insegnamento di Mazzarino si attuava nelle lezioni universitarie, attraverso i suoi scritti e, in grandissima parte, in luoghi non istituzionali. Trasferitosi a Roma, cercò disperatamente di riprodurre nella capitale l'antropologia del caffé siciliano, e l'impresa non era facile, se consideriamo che i locali romani erano (e in parte ancora sono) tra i più inospitali d'Europa. Comunque dava ai suoi allievi appuntamenti quasi sempre individuali - in questo o in quel bar $^{19}$, e in quelle lunghe conversazioni la storia romana aveva uno spazio esiguo. Fuori dell'università Mazzarino amava parlare

17 A. Ennout e A. Meillet, Dictionnaire étymologique de la langue latine, Paris, 1959, s.v. "disco", p. 176; ardua l'etimologia da discipio, per la quale A. Walde e J.B. Hofmann, Lateinisches Etymologisches Wörterbuch, I, Heidelberg, 1938, s.v. "discipulus”, p. 355. Sull'importanza del concetto di «fedeltà» nella sociologia accademica, F. WAQUET, Les enfants de Socrate..., p. 149.

19 Sull'importanza dei luoghi d'insegnamento diversi dall'università, F. WAQUET, Les enfants de Socrate..., p. 224-226. 
di altro, soprattutto di storia contemporanea e di attualità politica. Ma queste apparenti divagazioni erano un aspetto fondamentale del suo magistero, perché contenevano un invito costante all'anticonformismo, alla visione caleidoscopica di un medesimo fatto, alla possibilità, anche per un giovane, di un approccio originale all'attualità. In anni in cui molti ritenevano - e i giovani erano prontissimi a crederlo - che la storia fosse soprattutto una creazione delle masse («Tebe dalle Sette Porte, chi la costuì?»), Mazzarino invitava a non trascurare l'impatto delle singole personalità, e ci insegnava a far dialogare il plurale con il singolare. S'imponeva in quelle occasioni il «metodo» di Mazzarino, ovvero la forma della sua intelligenza.

Riflettere sul senso e sulla pratica del discepolato in una grande scuola universitaria significa suscitare immediatamente un altro dei quesiti posti da Anabases. se il rapporto con il mio maestro abbia conosciuto crisi o rotture. Poiché quel rapporto è durato circa vent'anni - dal 1967, anno in cui ho seguito il primo corso di storia romana all'Università La Sapienza, al 1987, anno della morte di Santo Mazzarino - è inevitabile che esso abbia attraversato momenti difficili, fasi di maggiore o di minore sintonia, sensazioni reciproche di delusione e di abbandono. Ma tutto ciò non ha avuto nulla a che fare con ragioni di carattere culturale e può essere quindi tenuto fuori dal discorso che mi è stato chiesto di svolgere. Ancor meno, in queste vicende, hanno pesato ragioni di carattere politico: quando sono diventato allievo di Santo Mazzarino avevo già i miei orientamenti, che erano grosso modo gli stessi del maestro, ma egli non cercò mai di indottrinare politicamente gli allievi e non selezionò mai né i giovani studiosi né gli amici in base a ragioni politiche (l'unica pregiudiziale, talmente ovvia da non essere esternata, riguardava l'estrema destra).

Poiché Mazzarino ha scritto su tutte le epoche della storia antica, e si è occupato di storia sociale, economica, monetaria, amministrativa, politica, culturale, religiosa, demografica, di storiografia antica e storiografia moderna, è difficile non riscontrare in moltissimi punti della mia produzione scientifica il diretto influsso del maestro: l'elenco sarebbe davvero interminabile. Poiché dispongo di una valutazione data da Mazzarino in una circostanza ufficiale, ho tuttavia la possibilità di riconoscermi, più in generale, nella sua valorizzazione di due aspetti principali, di ordine culturale, che a suo avviso qualificavano la mia appartenenza alla sua scuola romana: il rilievo dello studio e della critica filologica del materiale epigrafico, la ricerca sui processi di sviluppo della storia romana, con procedimenti in grado di individuare, fino alla tarda antichità, un intreccio peculiare di continuità e 'rivoluzioni'.

Sarebbe grave se nei miei lavori che trattano temi significativi nella produzione di Mazzarino, non avessi a mia volta trovato vie personali: difendo e rafforzo per esempio la datazione mazzariniana del de rebus bellicis ${ }^{20}$, ma non ritengo che le macchine

20 A. Giardina (a cura di), Anonimo, Le cose della guerra, Mondadori, Milano, 1989. 
descritte in quel trattato rappresentassero "un serio travaglio d'ingegneria militare ${ }^{21}$ »; preferisco invece inquadrarlo nella tradizione del macchinismo fantastico, destinata a una fortuna più che millenaria (evidente per esempio nello straordinario caso del carro falcato, di cui Voltaire fu accanito promotore). Ho seguito il maestro nella necessità di inquadrare sul lunghissimo periodo la storia del concetto di Italia ${ }^{22}$, ma non credo che l'idea di nazione sia applicabile alla storia dell'Italia, nemmeno temperata dall'avverbio «quasi ${ }^{23}$ ».

Sarebbe non meno grave se in circa quarant'anni di studio, in parallelo con le trasformazioni della storiografia e della cultura contemporanea, non avessi percorso a mia volta strade diverse, e non mi fossi occupato di temi che il mio maestro non ha affrontato o si è limitato a sfiorare. Per alcuni di questi ultimi - per esempio Silla, Seneca, Cassiodoro - mi riesce difficile individuare un preciso influsso: dobbiamo riconoscere che le attuali forme di organizzazione della cultura pongono in primo piano il ruolo della "committenza» esercitata da amici e da colleghi ${ }^{24}$, e ammettere al tempo stesso che le nostre scelte non hanno sempre una motivazione evidente, una ragione che risulti chiara a noi stessi. Ma accanto al maestro di cui sono stato discepolo ho avuto, com'era inevitabile, altri maestri: studiosi che ho conosciuto occasionalmente o non ho mai incontrato, e con i quali ho intrattenuto una grande familiarità libresca. Il mio debito nei confronti della storiografia francese è grandissimo e riguarda anche storici di altre epoche, come Fernand Braudel e soprattutto Jacques Le Goff. Ma in alcuni miei lavori dovrebbero essere evidenti le tracce dell'insegnamento proveniente della lettura di Peter Brown, Aron Ja. Gurevič, ed Eric Hobsbawm. Questi storici mi hanno suggerito l'interesse per la storia dei valori sociali, delle rappresentazioni, delle categorie culturali, oltre ad alcuni spunti antropologici. Per la storia del paesaggio italiano ho trovato il mio maestro in Emilio Sereni: «spazi aperti», «economia della selva» e altri concetti che hanno ispirato alcuni miei scritti mi sono stati suggeriti appunto da opere di Sereni (del quale ho anche avuto modo di studiare l'archivio depositato presso la Fondazione Istituto Gramsci) ${ }^{25}$. Per la storia dell'Italia romana ho invece tratto insegnamenti preziosi da due maestri con cui da molti anni ormai ho la fortuna di dialogare: Emilio Gabba, per il periodo repubblicano e il principato, Lellia Cracco Ruggini per l'età

21 S. MAZZARINo, Aspetti sociali del quarto secolo..., p. 62 (p. 74).

22 A. Giardina, L'Italia romana. Storie di un'identità incompiuta, Laterza, Roma-Bari, 1997.

23 S. MAZZARINo, Il pensiero storico classico..., II $^{1}$, cap. 10.

24 Senza la generosità di G. Cavallo (da cui ho appreso l'importanza della storia della scrittura come chiave di storia sociale) probabilmente non mi sarei mai occupato dell'età teodericiana (le mie ricerche si trovano ora in Cassiodoro politico, L'Erma di Bretschneider, Roma, 2006) o della storia del Caucaso ["Roma e il Caucaso" in Il Caucaso: cerniera fra culture dal Mediterraneo alla Persia (secoli IV-XI), Settimane di studio del Centro italiano di studi sull'alto Medioevo, Spoleto, 1996, p. 85-141].

25 A. Giardina, "Emilio Sereni e le aporie della storia d'Italia" (1996), poi in L'Italia romana..., cap. VIII ; questo interesse è stato incoraggiato dalla cultura e dall'entusiasmo di Luigi Capogrossi Colognesi. 
tardoantica. Da Silvio Panciera, mio professore di epigrafia alla Sapienza, ho appreso quanto sia arduo e al tempo stesso importante, per il futuro della storia antica, difendere lo statuto dell'epigrafia come «disciplina autonoma», scoraggiando al tempo stesso qualsiasi pretesa di autosufficienza ${ }^{26}$.

Oltre alla scuola che si riconosceva nel mio maestro Santo Mazzarino, ho avuto la possibilità di sperimentare un'altra «scuola» le cui attività si sono svolte, con il concorso di ricercatori e professori universitari, al di fuori dell'università. Per inquadrare questa esperienza è indispensabile tornare alla fine degli anni Sessanta. Ho ricordato quale fosse l'atmosfera dominante nei corsi di lettere classiche quando, nel 1966, ho incominciato a frequentarli. Ma con il Sessantotto quell'atmosfera fu travolta. Improvvisamente le materie antichistiche apparvero come armadi pieni di polvere e di ragnatele, mentre s'imponeva il primato politico e culturale della storia contemporanea: si teorizzava l'oblio e si sosteneva che l'unica storia degna di essere studiata da un giovane desideroso di dare il suo contributo al cambiamento della società fosse quella che coincideva con la nascita del movimento operaio o, al massimo, con la Rivoluzione francese. Le aule dove illustri docenti di cose antiche tenevano le loro lezioni si svuotarono da un giorno all'altro, mentre si denunciava la natura classista e reazionaria del liceo classico. Definirsi studente di lettere classiche suscitava un senso di colpa, una lieve vergogna, perché appariva quasi una dichiarazione d'insensibilità rispetto alla rivoluzione che si svolgeva sotto i nostri occhi. A chi era ancora in grado di orientare diversamente il proprio futuro, s'imponeva un'alternativa: dedicarsi ad altro oppure trovare nuove motivazioni.

Questa situazione di crisi dell'antichistica italiana trovò una risposta efficace e precoce nel gruppo della rivista Dialoghi di Archeologia, fondata nel 1967 da Ranuccio Bianchi Bandinelli. L'iniziativa, sorretta da forme di partecipazione e di organizzazione che non avevano alcun riscontro nel mondo accademico, mirava al superamento dell'archeologia intesa come disciplina tecnico-professionale mediante la sua costituzione come «ricerca storica» e una forte apertura interdisciplinare. In questa prospettiva, il lavoro di gruppo assumeva un chiaro significato strategico: «Per arrivare a concepire l'archeologia come ricerca storica e non come disciplina tecnico-professionale, occorre oggi che la collaborazione fra gli studiosi s'indirizzi sempre più verso indagini svolte effettivamente in comune ${ }^{27}$.»

26 Su questo punto rimando al mio intervento in M.L. Caldelli, G.L. Gregori e S. Orlandi (a cura di), Epigrafia 2006. Atti della XIV Rencontre sur l'épigraphie in onore di Silvio Panciera con altri contributi di colleghi, allievi e collaboratori, Quasar, Roma, 2008, p. 13-19.

27 La nascita del gruppo e della rivista è brevemente rievocata da R. BIANCHI BANDINELLI, AA., BB. AA. e BC. L'Italia storica e artistica allo sbaraglio, De Donato, Bari, 1974, p. 272-275. Sul contesto politico-culturale della vicenda, M. BARBANERA, Ranuccio Bianchi Bandinelli. Biografia ed epistolario di un grande archeologo, Skira, Ginevra-Milano, 2003, p. 347-350. 
Nel programma del gruppo dei Dialoghi di Archeologia, composto in massima parte da archeologi e da storici dell'arte antica, il rinnovamento della ricerca era presentato come inscindibile da un parallelo impegno a favore della tutela del patrimonio archeologico, artistico e paesistico ${ }^{28}$. In questo modo, l'impegno di studio degli antichisti di sinistra si saldava a un'importante lotta civile. Personalmente, ho vissuto solo ai margini e in ritardo l'esperienza dei Dialoghi di Archeologia, e spiego il fatto unicamente con motivi caratteriali (un'eccessiva timidezza che mi portava a guardare più che a partecipare). Ma era impossibile non trarre beneficio da quella nuova atmosfera.

In quegli anni la cultura antichistica italiana aveva bisogno non solo di trovare nuove motivazioni, ma anche - e le due esigenze s'intrecciavano - di democratizzare la propria immagine. Questa seconda operazione richiedeva anzitutto una critica della tradizione relativa al mondo classico nell'Occidente contemporaneo. Inevitabilmente questa critica assumeva le caratteristiche di una "autocritica», che si esprimeva, in modo particolare, nella ricostruzione del ruolo delle «ideologie del classicismo» sotto il fascismo e il nazismo. Di questi argomenti, nelle aule universitarie non si parlava. Questo silenzio non dipendeva tanto da un preciso orientamento politico, quanto dal rispetto di una concezione aulica dell'insegnamento, che disdegnava, in quanto non pertinenti e volgari, le contaminazioni politiche della scienza. Questa remora traspariva anche dalle formulazioni più illuminate e aperte: quando, in una rassegna datata Oxford 1945, Arnaldo Momigliano affermava che «il vero male fatto dal fascismo agli studi di storia antica» non stava «nelle sciocchezze che si dissero, ma nei pensieri che non furono più pensati ${ }^{29}$ ", implicitamente affermava l'irrilevanza culturale di quelle «sciocchezze», il loro essere non pertinenti alla storia degli studi. In Italia, un diverso orientamento, volto a una considerazione globale di quel rapporto e all'indagine dell'ideologia che lo sosteneva, è stato promosso da Luciano Canfora e dalla sua scuola ${ }^{30}$. Malgrado le opposizioni e le reazioni scandalizzate suscitate in alcuni settori del mondo accademico, l'operazione ha molto contribuito a favorire la circolazione dell'antichistica nel più ampio scenario della storiografia italiana. Grazie a essa, gli storici dell'antichità possono per altro rivendicare il merito di aver valorizzato per primi l'importanza del mito di

Per una panoramica sul rapporto tra antichistica e marxismo in quegli anni rinvio al mio, "Marxism and Historiography: Perspectives on Roman History", in CH. WickHAM (a cura di), Marxist History-writing for the Twenty-first Century (British Academy Occasional Paper, 9), Oxford University Press, Oxford, 2007, p. 15-31.

28 Cf. la "Presentazione" del primo numero (1967) della rivista, specialmente p. 5.

29 A. Momigliano, "Gli studi italiani di storia greca e romana dal 1895 al 1939" in Contributo alla storia degli studi classici, Roma, Edizioni di storia e letteratura, 1979, p. 296; per altro verso, Momigliano riconosceva che fascismo e nazismo ebbero «l'effetto positivo di incoraggiare ricerche su temi importanti prima trascurati», quali «le civiltà pre-romane d'Italia, il passaggio dalla repubblica all'impero, l'arte romana» (p. 292).

30 Cf. il dibattito "Per una discussione sul classicismo nell'età dell'imperialismo" aperto da L. CANFora sulla rivista da lui fondata: in particolare, Quaderni di storia 2 (197), 3 e 4 (1976), dello stesso autore, Le ideologie del classicismo, Torino, Einaudi, 1980. 
Roma nella storia politica del ventennio fascista e nella costruzione del consenso al regime $^{31}$.

Negli stessi anni, contribuiva notevolmente alla democratizzazione dell'antichistica la diffusione di una nuova filologia, rappresentata dalla studio della cultura materiale. In Italia, questa «rifondazione materialistica degli studi classici» è legata in particolare agli scritti di Andrea Carandini e dei suoi allievi ${ }^{32}$. Nozioni e metodi fino ad allora confinati nell'ambito ristretto di alcuni archeologi entravano ora in un dibattito culturale più ampio e concorrevano al superamento di quella poetica del capolavoro che aveva offerto autorevoli giustificazioni ai danni inferti al paesaggio urbano e rurale.

Nel volgere di pochi anni il panorama era dunque cambiato: sembrava ora possibile, ai giovani studiosi che si occupavano del mondo antico, superare la crisi d'identità aperta dal primato della politica e della contemporaneità e svolgere il proprio mestiere di antichisti in un modo più convinto, senza il timore di ritrovarsi in un'ansa protetta ma oscura, dignitosa ma superflua. La scossa del Sessantotto fu dunque salutare perché produsse un ripensamento del ruolo degli antichisti nella società contemporanea, pose su basi diverse il senso della loro funzione civile e politica, rinnovò i metodi della ricerca e suscitò nuove idee.

Si pose anche con forza, in quegli stessi anni, un'esigenza che è stata determinante nella mia decisione di non abbandonare il campo della storia antica: quella di non tenere l'interesse per il mondo antico confinato nelle aule universitarie e nelle biblioteche erudite ma di diffonderlo, cogliendo e promuovendo tutte le occasioni per una sua comunicazione più larga. In un paese come l'Italia dove, a differenza dei paesi di lingua inglese e della Francia, non esisteva alcuna tradizione in tal senso, l'impresa era tutt'altro che facile, ma ogni minimo successo dava grande soddisfazione ${ }^{33}$. Il discorso sulle scuole universitarie dovrebbe essere a questo punto allargato al mondo dell'editoria, che in alcune circostanze, in presenza di grandi personalità di editori (nel mio caso l'indimenticabile figura di Vito Laterza), ha rappresentato esso stesso una «scuola», un luogo di addestramento degli studiosi alla promozione delle idee, al dialogo tra competenze diverse, all'elaborazione di progetti, alla ricerca della scrittura adatta.

31 Sulla rimozione di questo tema nel panorama degli studi di storia contemporanea fino agli anni Settanta, ho dato qualche indicazione in A. Giardina [- A. Vauchez], Rome. L'idée et le mythe du Moyen Âge à nos jours, Fayard, Paris, 2000, p. 207-213.

32 Un ampio dibattito, non privo di aspre polemiche, fu suscitato dal suo Archeologia e cultura materiale, De Donato, Bari, 1975; per le reazioni, cf. l'Introduzione alla seconda edizione, ivi, 1979.

33 La mia ammirazione, già alta, per Jacques Le Goff ha avuto un'impennata quando, nel 1995, in occasione della presentazione dell'edizione italiana (Laterza, Roma-Bari, 1995) del suo L'Europe racontée aux jeunes, Seuil 1996, lo sentii affermare, davanti a una platea stupefatta, di aver concepito il suo mestiere di storico su tre piani - la ricerca, l'insegnamento e la divulgazione - e di averli considerati tutti e tre di pari dignità. 
Per la mia formazione di storico è stata molto importante, la partecipazione, presso l'Istituto Gramsci di Roma, ai lavori del «Seminario di Antichistica» fondato nel 1974 per iniziativa di Aldo Schiavone. L'Istituto godeva allora di un notevole prestigio per il contributo fornito alla politica culturale del partito, per la sua capacità d'intervento sui vari aspetti del rapporto tra intellettuali e politica, per il livello delle personalità che partecipavano ai suoi programmi, per l'importanza dei suoi archivi e per la ricchezza della sua biblioteca, aperta a un grandissimo numero di utenti. L'Istituto Gramsci era il simbolo dell'egemonia culturale comunista in Italia, e il fatto che esso offrisse spazi e risorse a un gruppo di antichisti poteva avere su questi ultimi un effetto alquanto rassicurante.

Il seminario degli antichisti attivo presso l'Istituto Gramsci rafforzava l'impronta interdisciplinare che aveva già caratterizzato il gruppo dei Dialoghi di Archeologia e ne conservava la struttura generazionale: esso raccoglieva infatti studiosi giovani, in massima parte trentenni, anche se non mancava l'apporto di professori già molto autorevoli (quali Antonio La Penna ed Ettore Lepore). I partecipanti erano studiosi dell'economia, del pensiero economico, della politica e della società, del diritto, della letteratura, dell'arte e della cultura materiale. Aveva tuttavia un interesse teorico molto più forte, che privilegiava lo studio delle riflessioni marxiane sulle società precapitalistiche ${ }^{34}$. Il lavoro teorico, tuttavia, non si esauriva in se stesso e mirava a stabilire un rapporto circolare con l'analisi erudita e con la critica delle fonti, in un progetto di ricerca concentrato su un unico grande tema: il modo di produzione schiavistico, interpretato come forma specifica, tipicamente romana, della schiavitù antica ${ }^{35}$. L'uso di un marxismo non dogmatico offriva inoltre possibilità più duttili di storicizzare le categorie moderne dell'economia politica, offrendo agli studiosi di cose antiche il vantaggio di prospettive precluse ad altri. Del resto la promozione di una conoscenza totale della storia era un'antica tradizione del pensiero marxista italiano, che affidava a questo aspetto la forza di analisi e di persuasione del «politico riformatore» nella società contemporanea.

Qui ho trovato una «scuola» diversa da quella universitaria. Il maestro di cui sono stato discepolo, come altri storici della sua generazione e della sua formazione, non amava esternare i fondamenti teorici del suo lavoro, pur avendone di solidissimi:

34 Il lavoro teorico del Seminario di Antichistica dell'Istituto Gramsci è stato pubblicato soprattutto in L. Capogrossi Colognesi, A. Giardina e A. Schiavone (a cura di), Analisi marxista e società antiche, Roma, Editori Riuniti, 1978; cf. A. CARANDINI, L'anatomia della scimmia. La formazione economica della società prima del capitale. Con un commento alle "Forme che precedono la produzione capitalistica» dai Grundrisse di Marx, Einaudi, Torino, 1979.

35 Le ricerche su questo tema sono poi confluite in A. Giardina e A. SChiavone (a cura di), Società romana e produzione schiavistica, 1-3, Roma-Bari, Laterza, 1981. 
era una questione di stile, una sorta di galateo erudito ${ }^{36}$. Egli era inoltre giustamente convinto che la formazione di un giovane studioso dovesse prevedere un lunghissimo apprendistato sulle fonti, e faceva propria un'espressione di spiriti niebuhriani cara a Walter Otto, suo professore all'università di Monaco nel 1936: ek mérous manthánomen, «impariamo dai particolari» (con il presupposto che l'imparare ek mérous comportasse l'impiego di energie ricostruttive e interpretative). Il suo insegnamento non prevedeva seminari collettivi e lavori di gruppo, mentre gli allievi si frammentavano nel dialogo con il maestro e non dialogavano fra loro (alcuni di noi avrebbero imparato a farlo in seguito). Come ogni grande storico dell'antichità, Mazzarino conosceva e adoperava documenti di vario genere, ma la preoccupazione di difendere l'«autonomia» della disciplina imponeva comportamenti cauti e senso della misura. In quell'altra scuola, grazie a «maestri» poco più grandi di me, in primo luogo Aldo Schiavone, mi sono addestrato alla fatica di comprendere linguaggi inusuali che non avevo appreso né al liceo né all'università, ho scoperto l'entusiasmo del lavoro di gruppo, ho verificato la possibilità di far convivere quell'acceso individualismo che è connaturato al nostro lavoro con la condivisione di un progetto comune.

Esprimere che cosa di tutto questo io abbia a mia volta saputo trasmettere, vorrebbe dire aprire un altro capitolo, che non tocca a me scrivere.

Andrea GiaRdina

Professeur d'histoire romaine

Istituto italiano di scienze umane

Palazzo Strozzi

Piazza degli Strozzi

50123 Firenze

andrea.giardina@sumitalia.it

36 Nel 1967 sentii parlare per la prima volta, in una lezione alla Sapienza, del concetto di «modo di produzione», ma si trattava di un professore allora ventottenne, Mario Liverani. 